\title{
UNINHIBITED NEUROGENIC BLADDER IN
}

ADULTS

ROBERT K. VINSON, M.D.

ANANIAS C. DIOKNO, M.D.

From the Section of Urology, Department of Surgery, University of Michigan Medical Center, Ann Arbor, Michigan

\begin{abstract}
The uninhibited neurogenic bladder is a common entity. It is characterized by urge incontinence, frequency, enuresis, and recurrent urinary tract infections. The diagnosis can only be made by a thorough urologic investigation, including cystometrography. A sound treatment program involves the use of anticholinergic medication to abolish uninhibited contractions, frequent voiding to keep intravesical pressure low, and antibiotic treatment when indicated for concurrent infection.
\end{abstract}

A practical classification of neurogenic bladder dysfunction, as introduced by McClellan ${ }^{1}$ and popularized by Nesbit and Lapides, ${ }^{2}$ has been essential to the renewed interest in the evaluation, diagnosis, and treatment of the neurogenic bladder. Disorders in neurovesical function are common problems; one of the most frequently encountered in medical practice is the uninhibited neurogenic bladder. To have a better understanding of this type of neurogenic bladder, a group of patients seen by the authors were reviewed. This article will present our observations on this group of patients.

\section{Method and Materials}

The charts of 64 adult patients with the diagnosis of uninhibited neurogenic bladder, confirmed by cystometry, were reviewed. A complete history and physical examination were performed in all patients. Diagnostic examinations included urine analysis and/or urine culture, excretory urography, and cystourethroscopy when indicated. Cystometry was performed in all patients using the water manometer apparatus. ${ }^{3}$

In this adult population the age range was between sixteen and eighty-five years: 3 patients were sixteen to nineteen years; 19 , twenty to thirty-nine; 18 , forty to fifty-nine; and 24 patients were over sixty years of age. The majority of patients were over forty years of age, and females (42 patients) outnumbered males (22 patients) almost two to one.

Presenting urologic complaints in the vast majority included urge incontinence ( $54 \mathrm{pa}$ tients), increased frequency of urination (55 patients), enuresis (34 patients), and past history of recurrent urinary tract infections (53 patients); 4 patients had urgency without incontinence and 4 had dysuria.

In 25 patients an obvious nervous system disorder was present: 9 patients had cerebrovascular

TABLE I. Cystometrographic findings in 64 patients

\begin{tabular}{lcccccc}
\hline & $\leqslant 50 \mathrm{ml}$. & $>50 \mathrm{ml}$. & $<100 \mathrm{ml}$. & $101-200 \mathrm{ml}$. & $201-300 \mathrm{ml}$. & $>300 \mathrm{ml}$. \\
\hline Residual urine & 54 & 10 & $\ldots$ & $\ldots$ & $\ldots$ & $\ldots$ \\
First urge to void & $\ldots$ & $\ldots$ & 22 & 37 & 5 & $\ldots$ \\
Volume at first contraction & $\ldots$ & $\ldots$ & 15 & 40 & 9 &. \\
Capacity & $\cdots$ &. & 1 & 22 & 33 & 8 \\
\hline
\end{tabular}


accidents; 8, multiple sclerosis; 4, Parkinson's disease; and 4 miscellaneous disorders (seizure, organic brain syndrome), but it is notable that in the remaining 61 per cent ( 39 patients) no neurologic abnormality could be detected.

Urinalysis at the time of evaluation revealed the majority of patients to have infected urine (52 patients). Excretory urograms were abnormal in 19 patients: 8 patients had evidence of chronic pyelonephritis, 6 had hydronephrosis, and 5 had renal or ureteral calculi.

Cystometric examination demonstrated in most patients a small capacity bladder with minimal residual urine. The onset of involuntary detrusor contractions was observed to occur within the first $200 \mathrm{ml}$. of bladder volume in 55 patients (Table I).

Forty patients were tested with $50 \mathrm{mg}$. of intravenous propantheline (Pro-Banthine) to abolish the uninhibited contractions and confirm the diagnosis of uninhibited neurogenic bladder.

Cystourethroscopy was performed in 54 patients. Findings were normal in 22 patients. Bladder trabeculation (32 patients) and chronic cystitis (23 patients) were the most common abnormal findings, and 5 patients had prostatic hypertrophy.

Sixty patients were treated with a regimen of frequent voiding (every two to three hours) and oral anticholinergic medication such as tincture of belladonna, 10 to 25 drops four times a day; or oxybutynin tablets $5 \mathrm{mg}$. three times a day. A short course of antibiotic therapy was prescribed in the majority of patients when indicated, but in several patients with long-standing chronic cystitis and bladder trabeculation, chronic lowdose antibiotics were used.

Follow-up has ranged from three months to three years. In 81 per cent excellent results were obtained, noted by the absence of urgency, incontinence, and recurrent urinary tract infection. The remaining patients had only partial response to therapy. This may represent a group of patients who would benefit from selective sacral neurectomy, as proposed by Susset et al. ${ }^{4}$ and Torrens and Griffith. ${ }^{5}$

\section{Comment}

The uninhibited ncurogenic bladder or infantile bladder is characterized by uncontrolled contractions of bladder smooth muscle. In the infant up to two to three years of age, filling of the bladder stimulates proprioceptive endings in the bladder wall which in turn elicit a micturition reflex and resultant detrusor contraction. With concomitant relaxation of the periurethral striated muscle, there is prompt emptying of the bladder at relatively low intravesical pressure. With maturation of the corticoregulatory tract, voluntary control of the urinary bladder occurs. In the adult an acquired defect in the corticoregulatory tract may result in an uninhibited bladder. It has been suggested that the persistence of the infant bladder in some adults is the result of incomplete development of the corticoregulatory tract governing micturition.

The majority of our patients had a past history of recurrent urinary tract infections which was not prevented with the usual modes of therapy (antibiotics, urethral dilatation, antispasmodics, and so forth).

In a series of 250 women with recurrent urinary tract infections, Lapides and Costello ${ }^{6}$ found 16 per cent to have uninhibited bladders. In another series of 71 girls the diagnosis of uninhibited neurogenic bladder was made in 60 per cent. $^{7}$

It has been shown that significant elevation of intravesical pressure leads to decreased blood flow to the bladder wall. ${ }^{8}$ This then leads to bladder wall ischemia and decreased host resistance to bacterial invasion. The increased intravesical pressure is caused by voluntary contraction of the periurethral striated muscle during an episode of uncontrolled detrusor contraction in an attempt by the patient to prevent incontinence or avoid voiding.

In the minority of our patients ( 25 of 64 ) defects in the corticoregulatory system could be readily explained by an obvious nervous system disorder. A representative example is a thirty-sixyear-old male who sustained a mild, left cerebrovascular accident secondary to malignant hypertension. Urologic symptoms of urgency, enuresis, frequency, and incontinence developed shortly after the stroke. However, in the majority of cases, no neurologic deficit was apparent, and the diagnosis of uninhibited neurogenic bladder could only be made by pertinent history and cystometrography.

A fifty-seven-year-old female was completely well until she had an acute onset of urge incontinence, frequency, and enuresis. Symptoms responded to frequent voiding and anticholinergic medications. The foregoing example is typical of several of our female patients and certainly deserves careful follow-up. There is a distinct possibility that an acute presentation of uninhibited detrusor contractions in an otherwise 
normal individual may represent the first signs of a nervous system disorder (namely, multiple sclerosis, brain tumor, and so forth).

\section{Ann Arbor, Michigan 48104 \\ (DR. DIOKNO)}

\section{References}

1. McClellan, F. C.: The Neurogenic Bladder, Springfield, Illinois: Charles C Thomas, 1939.

2. Nesbit, R. M., Lapides, J., and Baum, W. C.: Fundamentals of Urology, Ann Arbor, Michigan, J. W. Edwards, 1953, p. 110.

3. LApides, J.: Cystometry. J.A.M.A. 201: 124 (1967).
4. Susset, J. G., et al.: Phenolisation et neurotomie selective dans le traitement de la dysfonction vesicale neurogene por lesion centrale incomplete, J. d'Urol. Nephrol. (Paris) (Suppl.) 75: 502 (1969).

5. Torrens, M. J., and Griffith, H. B.: The control of the uninhibited bladder by selective sacral neurectomy, Br. J. Urol. 46: 639 (1974).

6. LAPIDES, J., and Costello, R. T.: Uninhibited neurogenic bladder: a common cause for recurrent urinary infection in normal women, J. Urol. 101: 539 (1969).

7. Lapides, J., and Diokno, A. C.: Persistence of the infant bladder as a cause for urinary infection in girls, Trans. Am. Assoc. Genitourin. Surg. 61: 51 (1969).

8. LAPIDES, J., and FinkBeiner, A.: Effect of distention on blood flow in dog's urinary bladder, Invest. Urol. 12: 210 (1974). 